\title{
Unusual initial presentation of ABPA as hydropneumothorax
}

\author{
Juvva Kishan Srikanth, Nitesh Gupta, Sumita Agrawal, Shibdas Chakrabarti, Pranav Ish
}

Department of Pulmonary, Critical Care \& Sleep Medicine, VMMC \& Safdarjung Hospital, New Delhi, India

A 48-year-old female with bronchial asthma presented with right-sided pleuritic chest pain since 20 days followed by cough with expectoration and shortness of breath since 15 days. She was not compliant with her asthma medications. On examination, the patient was febrile with tachycardia, tachypnea, hypotension and room air saturation of $85 \%$. Respiratory system examination revealed tracheal deviation to the left, reduced chest expansion and decreased breath sounds in the entire right hemithorax and the presence of succussion splash.

Chest X-ray PA view showed right hydropneumothorax. High-resolution computed tomography (HRCT) scan of the thorax demonstrated right-sided loculated hydropneumothorax with collapse of the underlying lung (Figure 1A). Intercostal drainage (ICD) tube insertion was performed and the pleural fluid aspirate showed exudative, lymphocytic fluid with low ADA. The patient was initiated on broad-spectrum antibiotic therapy. Follow-up HRCT scan demonstrated complete resolution of hydropneumothorax, and ICD tube was removed. Also, the presence of underlying bronchiectasis (varicose type) was documented on the right side (Figure 1B). On further evaluation the patient was found to have peripheral eosinophilia (A.E.C. $=3800 / \mu \mathrm{L}$ ). The Af-IgE $(0.58 \mathrm{kU} / \mathrm{L})$, total IgE (2157 IU/mL) and Af-IgG (141 mgA/L) were positive; thereby confirming allergic bronchopulmonary aspergillosis (ABPA) as per ISHAM criteria [1]. Alternative etiologies of hydropneumothorax were ruled out by negative sputum and pleural fluid analysis for tubercular, bacterial and fungal cultures.

The patient was initiated on inhaled therapy and oral Prednisolone at a dose of $0.5 \mathrm{mg} / \mathrm{kg} / \mathrm{day}$ for two weeks, then on alternate days for eight weeks [1]. The woman improved symptomatically after 10 weeks and is currently under follow-up.

The literature search yielded 7 case reports of pneumothorax/hydropneumothorax in ABPA [2-5]. Of 7 cases, only 3 had initial presentation as pneumothorax/hydropneumothorax [2-4]; the remaining four had pleural complication on follow-up. The probable etiology of pleural complications in ABPA is either secondary to severe underlying bullous lung disease or impacted mucus acting as a ball valve leading to air trapping and rupture of the bronchus [2-5].

This case highlights the need to suspect ABPA, even if the patient presents with pleural pathology (pneumothorax or hydropneumothorax) in a known case of bronchial asthma and peripheral eosinophilia.
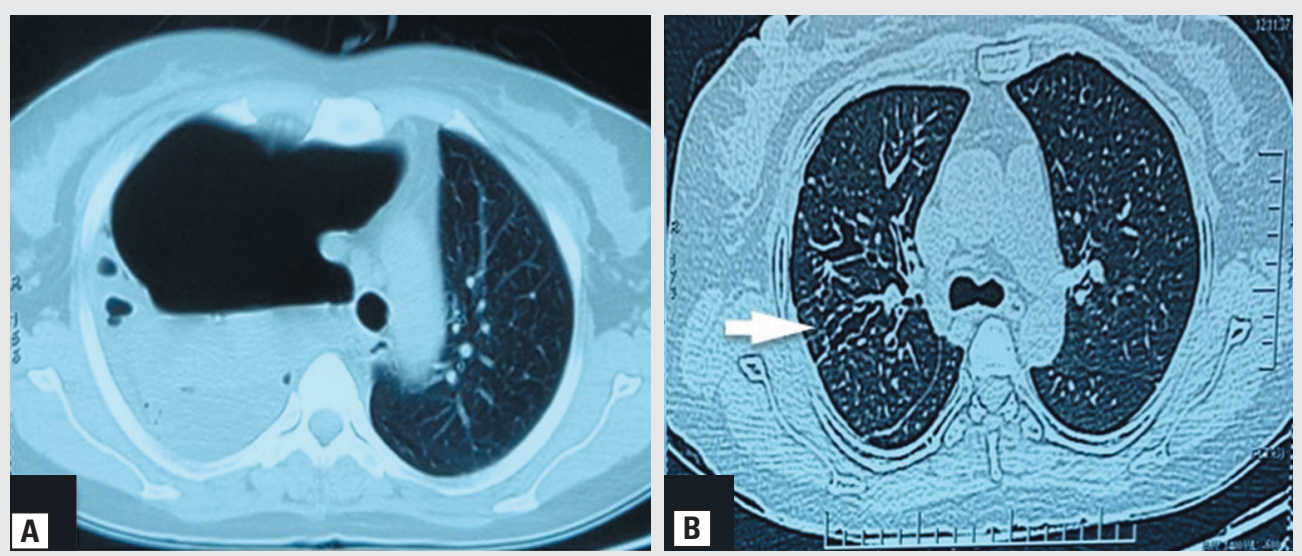

Figure 1. A. High-Resolution Computed Tomography of the chest demonstrating right-sided hydropneumothorax; B. complete re-expansion of the right lung with the presence of central bronchiectasis (varicose type; white arrow)

Address for correspondence: Nitesh Gupta, Department of Pulmonary, Critical Care \& Sleep Medicine, VMMC \& Safdarjung Hospital, New Delhi, India;

e-mail: niteshgupta2107@gmail.com

DOI: 10.5603/ARM.2020.0115

Received: 20.01.2020

Copyright (C) 2020 PTChP

ISSN 2451-4934

Conflict of interest: None declared 


\section{References:}

1. Agarwal R, Chakrabarti A, Shah A, et al. For the ABPA complicating asthma ISHAM working group. Clinical \& Experimental Allergy. 2013; 43: 850-873.

2. Das A, Das S, Basuthakur S. Right-sided pneumothorax and right upper lobe collapse: Two unusual presentations of allergic bronchopulmonary aspergillosis. The Journal of Association of Chest Physicians. 2014; 2(2): 71, doi: 10.4103/2320-8775.135116.

3. Vishnukanth G, Dwivedi DP, Joseph J, et al. Secondary spontaneous pneumothorax. An unusual clinical presentation of allergic bronchopulmonary aspergillosis. Indian Journal of Immunology and Respiratory Medicine. 2017; 2(3): 93-95.

4. Suri T, Makkar N, Ray A, et al. A unique case of hydropneumothorax in allergic bronchopulmonary aspergillosis. Med Mycol Case Rep. 2019; 25: 29-31, doi: 10.1016/j.mmcr.2019.07.003, indexed in Pubmed: 31338287.

5. Isaac BT, Samuel JT, Mukherjee DK, et al. Loculated pneumothorax due to a rare combination resulting in an interesting chest radiograph. Clin Respir J. 2017; 11(6): 1074-1078, doi: 10.1111/cri.12457, indexed in Pubmed: 26789278. 